\title{
DETECTION OF RANDOM TARGETS IN SENSOR NETWORKS WITH APPLICATIONS ${ }^{1}$
}

\author{
Minyi Huang*, Girish N. Nair* \\ ${ }^{*}$ Dept. Electrical and Electronic Engineering, University of \\ Melbourne, Parkville, 3010 Victoria, Australia.
}

\begin{abstract}
This paper considers the detection of random targets modelled by a hidden Markov chain using distributed multiple sensors. The optimization problem is formulated as an optimal stopping problem which intends to increase the capturing probability of the moving object. The general formulation in this paper may be applied for distributed (change or failure) detection in a networked system, which generalizes the traditional quickest detection. Copyright (c)2005 IFAC
\end{abstract}

Keywords: Hidden Markov models, sensor networks, optimal stopping, target detection, distributed change detection.

\section{INTRODUCTION}

Recently there is a rapidly increasing interest in detection and tracking of targets in sensor networks due to its wide application background ranging from location awareness services and intrusion detection to certain military applications, etc.; see (Aslam et al., 2003; Chong and $\mathrm{Ku}$ mar, 2003; Zhao et al., 2003).

This paper investigates the detection of a random target in a distributed network in which a set of sensor nodes are geographically distributed and each connected to a fusion center by a wireless channel. At each node, due to its limited computational capability, storage capacity, and communication bandwidth constraints, the sensor needs to form a local decision (to be called a message) to send to the fusion center where final information processing takes place.

Suppose the target's state (e.g. location) is modelled by a finite state Markov chain (a simple example being a one or two dimensional random walk for motion modelling), and is measured by sensors in its vicinity with additive noise. The

\footnotetext{
1 This work was partially supported by ARC.
}

fusion center needs to combine the messages received from all sensors to infer the state of the target. In this paper, our interest is in obtaining accurate instantaneous state estimation at the fusion center. This differs from the sate estimation problem considered in the companion work (Huang and Dey, 2005). This kind of objective is well motivated by certain applications, for instance, the interception of a hostile target (Burr et al., 1985). In such scenarios, it is important to have reliable knowledge for the target state at a certain time stage, rather than have good estimation averaged over time. Furthermore, once the fusion center is convinced of the state, for instance the location, of the target, it needs to communicate with a corresponding local unit to take action (e.g. fire for interception), or it simply sends out a warning message to that local region. Thus the network performance relies on the correctness of its decision and the associated local action, and further operation of the system following this pair of decision and action is not of primary interest. This is in contrast to the hierarchical algorithm proposed in (Oh et al., 2005) for estimating the number and position of multiple targets on a specified period. 
Based on the above justification, the optimization problem is formulated as an optimal stopping problem where the performance measure is related to (1) system operating cost, (2) action cost and (3) miss penalty. Notice that here the quantization scheme for the sensors is part of the optimization problem. Such a model may be regarded as a special networked control system where the fusion center is equivalent to a controller or decision maker which is equipped with high computing resources and may command the actions of the actuator (e.g. intercepter) associated with each sensor node at the local area.

In our analysis the stopping problem is converted into an optimal control problem for which the solution is computed by the dynamic programming equation. The state set for the stopping action is specified in terms of the information state. It is also shown that there is a separation between the quantization optimization and the determination of the stopping action. Finally, based on the general formulation in this paper, an application example is considered for distributed change or failure detection in a network. This generalizes the traditional Bayesian quickest detection problem studied by many authors; see, e.g., (Shiryayev, 1978; Kailath and Poor, 1998).

\section{THE SYSTEM MODEL}

To begin with, the basic infrastructure of the network is specified as follows. First, assume $n$ sensor nodes are placed at different sites, each monitoring its surrounding region to be called a cell. The $n$ cells are assumed to be disjoint. Second, the motion of the target is modelled by a finite state Markov chain $X \triangleq\left\{X_{t}, t \geq 1\right\}$ with state space $S=\left\{s_{1}, \cdots, s_{n}, s_{n+1}\right\}$. Let $I_{n}=$ $\{1, \cdots, n\}$, and $I_{n+1}=\{1, \cdots, n, n+1\}$. The state $X_{t}=s_{i}, i \in I_{n}$ at time $t$ means that the target is in cell $i$. The target may transit from one cell to another. Here the extra state $s_{n+1}$ denotes an outer site which is beyond the network's surveillance coverage. The target may arrive from or leave for the outer site. This is a natural scenario for many practical situations. The transition probability matrix of $X$ is given as $P \in \mathbb{R}^{(n+1) \times(n+1)}$.

\subsection{The measurement model}

At time $t \geq 1$, node $i$ has a noisy measurement $Y_{i, t}$ which depends on the current state of $X$. When the target is in its cell, the measurement has a high signal to interference ratio, and otherwise, only an attenuated or even negligible signal concerning the target is reflected. Thus, the set of $n$ nodes

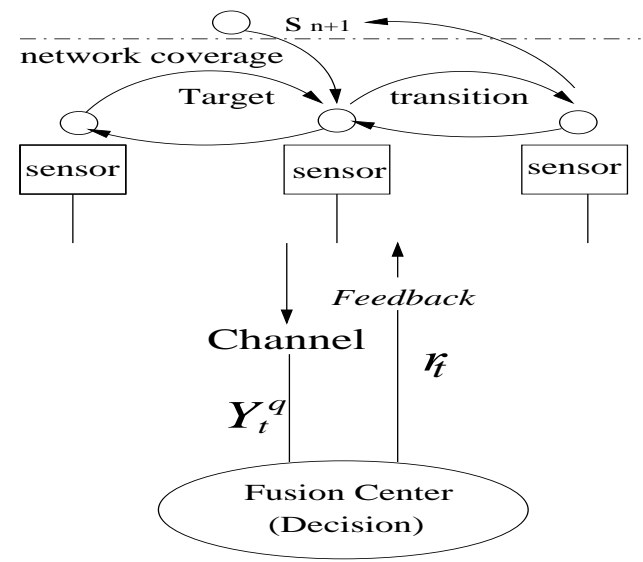

Fig. 1. A sensor network with feedback for dynamic quantization. The site associated with $s_{n+1}$ is beyond coverage.

produces location dependent information for the target. The output equation is given as

$$
Y_{i, t}=f_{i}\left(X_{t}\right)+W_{i, t}
$$

where $f_{i}\left(s_{n+1}\right)=0$ for all $i \in I_{n}$, i.e., for $X_{t}=$ $s_{n+1}$ only the background noise is obtained due to the limitation of network coverage.

To simplify the analysis and specify the location dependent observations, it is assumed that the sites are aligned along a line, and in addition that the target has negligible effect on more distant sensors, i.e., the target only affects the measurements of its visiting and immediate neighboring cells. The generalization of our analysis to the more general network topology is straightforward.

Now the measurement can be written as

$$
Y_{i, t}=\sum_{j=1}^{n} F_{i j} 1_{\left(X_{t}=s_{j}\right)}+W_{i, t}
$$

where the matrix $F=\left(F_{i j}\right)$ is a tridiagonal matrix

$$
\left[\begin{array}{ccccc}
f_{1}(1) & f_{1}(2) & 0 & \cdots & 0 \\
f_{2}(1) & f_{2}(2) & f_{2}(3) & \cdots & 0 \\
\cdots & & & & \cdots \\
0 & \cdots & \cdots & f_{n-1}(n-1) & f_{n-1}(n) \\
0 & \cdots & 0 & f_{n}(n-1) & f_{n}(n)
\end{array}\right]
$$

where $f_{k}(i) \triangleq f_{k}\left(s_{i}\right)$ for $i \in I_{n}$. The above equation may be written in the vector form

$$
Y_{t}=F \operatorname{Diag}\left(1_{\left(X_{t}=s_{i}\right)}\right)_{n} 1_{n}+W_{t}
$$

where $Y_{t}=\left[Y_{1, t}, \cdots, Y_{n, t}\right]^{T}, 1_{n}$ is a column vector with $n$ entries being 1 , and $W_{t}=\left[W_{1, t}, \cdots, W_{n, t}\right]^{T}$. The noise $\left\{W_{t}\right\}$ is assumed to be a sequence of i.i.d. random variables taking values in $\mathbb{R}^{n}$.

\subsection{The dynamic binary quantization}

In order to reduce the communication capacity requirement of each node, the binary quantization scheme is adopted which, in turn, is denoted by a 
parameter $r_{i, t}$ partitioning the range space of $Y_{i, t}$, $i \in I_{n}, t \geq 1$. Each sensor needs to send a symbol in a set of two entries to the fusion center based on its own measurement. Without losing generality one can use the same alphabet set $\left\{a_{1}, a_{2}\right\}$ with two distinct entries for all sensors. However, now a static quantization scheme is inadequate for two reasons. On one hand, unlike the usual hypothesis testing as examined in (Chamberland and Veeravalli, 2003), here the distribution of the underlying measurements evolves in a dynamic manner as driven by the Markov chain; on the other hand, the super-diagonal and subdiagonal entries in $F$ make the signal discrepancy associated with neighboring cells further blurred. Hence in the paper a dynamic binary quantization scheme is adopted. By doing so the local node can optimize its quantization threshold by following the command of the fusion center so that refined information can be conveyed to the latter.

Now, for a given quantization parameter $r_{i, t}$ for the $i$-th sensor at time $t$, the resulting output is

$$
Y_{i, t}^{q}= \begin{cases}a_{1} & Y_{i, t}<r_{i, t} \\ a_{2} & Y_{i, t} \geq r_{i, t}\end{cases}
$$

$Y_{t}^{q}=\left[Y_{1, t}^{q}, \cdots, Y_{n, t}^{q}\right]^{T}$ shall be called a message to be received by the fusion center. For optimizing the quantizer $r_{t}=\left(r_{i, t}\right)_{n}$, a set $S_{t h}$ of threshold candidates is selected first. Since the threshold needs to be set by the command from the fusion center via the inverse channel, it is difficult to implement and optimize the quantization by choosing $S_{t h}$ as a continuum. Instead, a discrete subset $L_{d}$ is used with corresponding components located between 0 and $f_{i}(i)$. Here it is assumed that $\left|f_{i}(i)\right|>\left|f_{i}(i-1)\right|,\left|f_{i}(i)\right|>\left|f_{i}(i+1)\right|$ in (1), and the physical interpretation is clear.

\subsection{The performance measure}

The performance function to be employed is the expectation of the cumulative sum of instantaneous costs $c\left(X_{t}, u_{t}\right)$ until a terminal time when the fusion center makes a final decision as to which cell contains the target and sends a reaction (e.g. fire) command to be executed instantly at that cell. Since at any time at most one node is instructed to react, a control variable for the set of all nodes can be introduced as a vector $u=(0, \cdots, 0)^{T} \triangleq e_{0}^{u}$ containing all zeros or $u=e_{i}^{u}, 1 \leq i \leq n$, where $e_{i}^{u}$ has all zero entries except the $i$-th being 1 . Here $e_{i}^{u}$ dictates that the $i$-th node takes reaction. If the $k$-th entry in $u$ is zero, it means the $k$-th node is in an idle state. In our formulation, the stopping time comes once a reaction is taken at one node. Let $u_{t}$ denote the control at time $t$.

Let us introduce the one stage cost:
- If $X_{t}=s_{i}, i \in I_{n+1}$ and $u_{t}=e_{0}^{u}$, this incurs a one step operating $\operatorname{cost} c\left(X_{t}, u_{t}\right)=c_{0}(i)>0$, and the system will evolve to next stage;

- If $X_{t}=s_{i}, i \in I_{n}$ and $u_{t}=e_{i}^{u}$, there is a reaction cost $c\left(X_{t}, u_{t}\right)=c_{1}(i) \geq 0$, and the system terminates;

- If $X_{t}=i, i \in I_{n+1}$ and $u_{t}=e_{j}^{u}$ such that $j \neq i$, the system terminates with a miss penalty $c\left(X_{t}, u_{t}\right)=c_{2}>0$.

The above specification clearly defines a cost function $c: S \times U \rightarrow \mathbb{R}^{+}$where $U=\left\{e_{0}^{u}, e_{1}^{u}, \cdots, e_{n}^{u}\right\}$. Write $c\left(s_{i}, u\right)=c(i, u), i \in I_{n+1}$. The motivation to introduce the state dependent cost term $c_{1}$ is that in a practical situation, the reaction units at different cells may function with different performance and associated expenses. Such a heterogenous cost measure may be used to encourage the deployment of reactions at certain locations.

As an example, one may take $c_{0}(1)=\cdots=$ $c_{0}(n)>c_{0}(n+1) \geq 0$ to indicate a penalty caused by the presence of target threat. Also, a large $c_{2}$ makes the fusion center more cautious in requesting a reaction; in other words, the fusion center tends to achieve a higher probability for capturing the target since the operating cost is dominated by the penalty for miss.

It is assumed throughout this paper that $c_{2}>$ $\max c_{0}(i)$. In the case $\max \left\{c_{2}, c_{1}(i), i \in I_{n}\right\} \leq$ $\min c_{0}(i)$, it is optimal to react at the initial time.

Remark: This paper only considers optimization based on "one shot". It is possible to consider the more general cases from the following aspects: (1) successive reactions (fires) and (2) probabilistic target hitting. To maintain a reasonable length, this paper shall not pursue these generalizations.

\subsection{The augmented controlled Markov process}

To apply dynamic programming, the above stopping problem will be converted into a Markov decision problem on the horizon $[0, \infty)$. Associated with $X$, let us construct an auxiliary controlled process $X^{o}$ which has state space $\{1, \cdots, n+2\}$ and controlled transition matrix

$$
\begin{aligned}
\left.P\right|_{u=e_{0}^{u}} & =\left[\begin{array}{cc}
P & 0_{(n+1) \times 1} \\
0_{1 \times(n+1)} & 1
\end{array}\right], \\
\left.P\right|_{u=e_{i}^{u}} & =\left[\begin{array}{cc}
0_{(n+1)} & 1_{(n+1) \times 1} \\
0_{1 \times(n+1)} & 1
\end{array}\right], i \in I_{n}
\end{aligned}
$$

where $P \in \mathbb{R}^{(n+1) \times(n+1)}$ is the transition matrix of $X$. For $X^{o}$, define the cost $c^{o}\left(s_{i}, u\right)=c(i, u)$ for $i \in I_{n+1}$, and $c^{o}\left(s_{n+2}, u\right)=0$, where $u \in U$. Essentially the controlled Markov chain $X^{o}$ is obtained by augmenting the state space of $X$ by a fictitious absorbing state $s_{n+2}$ such that the state of $X^{o}$ would transit from $s_{1}, \cdots, s_{n+1}$ to $s_{n+2}$ once an action $e_{i}^{u}, i \in I_{n}$ is taken. Let the 
observation of $X^{o}$ be specified by (2) using the same output variable $Y$ and replacing $X$ by $X^{o}$. If $X^{o}=s_{n+2}$, it is assumed that all $Y_{i}^{q}, i \in I_{n}$, take $a_{1}$. The output of $Y_{i}^{q}$ corresponding to $s_{n+2}$ does not affect the optimal stopping rule associated with the original Markov chain $X$.

See (Bertsekas, 1995) on converting an optimal stopping problem into an optimal control problem with an infinite horizon. In addition, once an optimal control law for $X^{o}$ is obtained, it is straightforward to retrieve the control law for $X$. Hence in the following it is sufficient to analyze in terms of $X^{o}$. For notational brevity, in the rest of the paper $X^{o}$ is simply written as $X$ but indicated at various places as a controlled process with state space $S=\left\{s_{1}, \cdots, s_{n+2}\right\}$. Also, omitting the superscript, $c(i, u)$ is used instead of $c^{o}(i, u)$ to denote the cost associated with the $n+2$ states.

\section{THE OPTIMAL CONTROL PROBLEM}

Denote by $Y^{q}$ the message received by the fusion center from all $n$ nodes. The control $u_{t-1}$ and quantizer $r_{t} \in \mathbb{R}^{n}$ for the $n+2$ state Markov chain are adapted to $\mathcal{F}_{t-1} \triangleq \mathcal{F}\left(Y_{i}^{q}, i \leq t-1\right)$ which is the $\sigma$-algebra generated by past messages. $\mathcal{F}_{0} \triangleq$ $\{\varnothing, \Omega\}$. In further analysis, a sufficient statistic will be recursively calculated such that the parameter $r_{t}=\left(r_{i, t}\right)_{n}$ need not be determined using the overall history $\left(Y_{1}^{q}, \cdots, Y_{t-1}^{q}\right)$ when the sufficient statistic is computed at each step. In this setup, the fusion center feeds back $r_{t}$ to the sensor nodes between the time instant $t-1$ and $t$, and at time $t$, the sensors employ $r_{t}$ for quantization resetting.

Let $I_{n+2}=\{1, \cdots, n+1, n+2\}$. Before formulating the stochastic control problem, it is necessary to introduce the so-called information state $\theta_{t}=\left[\theta_{1, t}, \cdots, \theta_{n+2, t}\right]^{T}$, where

$$
\theta_{i, t}=E\left[1_{\left(X_{t}=s_{i}\right)} \mid \mathcal{F}_{t}\right], \quad i \in I_{n+2}, \quad t \geq 1 .
$$

The recursion for the information state is given as

$$
\begin{aligned}
\theta_{t+1} & =\frac{1}{z_{t+1}} Q\left(s_{1}, \cdots, s_{n+2}, r_{t+1}, Y_{t+1}^{q}\right)\left(\left.P\right|_{u}\right)^{T} \theta_{t} \\
& \triangleq \frac{1}{z_{t+1}} T\left(s_{1}, \cdots, s_{n+2}, r_{t+1}, Y_{t+1}^{q}\right) \theta_{t}
\end{aligned}
$$

where $\left.P\right|_{u}$ is the transition matrix of $X_{t}$, and $z_{t+1}$ is a normalizing factor such that $\left|\theta_{t+1}\right|=1$, where $|x| \triangleq \sum_{i=1}^{n+2}\left|x_{i}\right|$ for $x \in \mathbb{R}^{n+2}$. The matrix $Q\left(s_{1}, \cdots, s_{n+2}, r_{t}, y_{t}^{q}\right)$ can be determined as

$$
Q(\cdot)=\left(\begin{array}{ccc}
F\left(s_{1}, r_{t}, y_{t}^{q}\right) & & \\
& \cdots & \\
& & F\left(s_{n+2}, r_{t}, y_{t}^{q}\right)
\end{array}\right),
$$

where $y_{t}^{q} \in \mathbb{R}$ denotes a value for $Y_{t}^{q}$. Here $F\left(s_{i}, r_{t},\left(a_{i_{1}}, \cdots, a_{i_{n}}\right)\right)=\int_{\mathcal{A}\left(r_{t}, y_{t}^{q}\right)} f\left(y_{1}-s_{i}, \cdots, y_{n}-\right.$ $\left.s_{i}\right) d y_{1} \cdots d y_{n}$ with $\mathcal{A}\left(r_{t}, y_{t}^{q}\right) \triangleq\left\{y \in \mathbb{R}^{M}, \mathcal{Q}\left(r_{t}, y\right)=\right.$ $\left.\left(a_{i_{1}}, \cdots, a_{i_{n}}\right)=y_{t}^{q}\right\}$, where $f$ is the joint probability density of the noise $W=\left(W_{1, t}, \cdots, W_{n, t}\right)^{T}$ and $\mathcal{Q}$ is defined in an obvious manner as the quantizer function associated with $r_{t}$.

Denote the range space for $\theta_{t}$ by the simplex

$$
\mathcal{D}=\left\{x \in \mathbb{R}_{+}^{n+2},|x|=1\right\} .
$$

Define the subset $\mathcal{D}_{0}=\left\{x \in \mathcal{D}, x_{n+2}=0\right\}$. If $\theta_{1} \in \mathcal{D}_{0}, \theta_{t}$ will stay in $\mathcal{D}_{0}$ until a stopping action $e_{i}^{u}, i \in I_{n}$ is taken at time $k$. To solve the stopping problem for the original $(n+1)$ state Markov chain, it suffices to find here a solution restricted to $\mathcal{D}_{0}$ for the case of $(n+2)$ states.

A sequence of controls $\left\{u_{t}\right\}$ (simply written as $u$ ) is said to be admissible if each $u_{t}$ is adapted to $\mathcal{F}_{t}$. We may use $r$ to denote the sequence $\left\{r_{t}, t \geq 1\right\}$ or simply a value in $\mathbb{R}^{n}$. Define the optimal cost, or value function to the completely observed optimal control problem (in terms of the information state) as

$$
v(\theta)=\inf _{r_{t}, u_{t}} E\left[\sum_{t=1}^{\infty} \theta_{t}^{T} c\left(\cdot, u_{t}\right) \mid \theta_{1}=\theta\right]=\inf _{u, r} J(\theta, u) .
$$

The dynamic programming equation is given as

$$
\begin{aligned}
& v(\theta) \\
= & \min _{r, u}\left[\theta^{T} c(\cdot, u)+\sum_{Y^{q}}\left|T\left(r, Y^{q}, u\right) \theta\right| v\left(\frac{T\left(r, Y^{q}, u\right) \theta}{\left|T\left(r, Y^{q}, u\right) \theta\right|}\right)\right] \\
\triangleq & \min _{r, u} H(r, u, \theta)
\end{aligned}
$$

where the cost $c(\cdot, u)$ is an $\mathbb{R}^{n+2}$ vector for $u \in$ $\left\{e_{0}^{u}, e_{1}^{u}, \cdots, e_{n}^{u}\right\}$ and $r$ is chosen from the finite set $L_{d} \subset \mathbb{R}^{n}$.

\section{SEPARATION OF QUANTIZATION AND ACTION REGION PARTITION}

Let us introduce the assumption:

(H1) The transition matrix $P$ of $X_{t}$ ( with $n+1$ states) is ergodic and in (5) each $F\left(s_{i}, r_{t}, y_{t}^{q}\right)>0$ for all $r_{t} \in L_{d}$ and $y_{t}^{q} \in\left\{a_{1}, a_{2}\right\}^{n}$.

Proposition 1. Under $(\mathrm{H} 1)$ and restricted to $\mathcal{D}_{0}$ the value function $v: \mathcal{D}_{0} \rightarrow \mathbb{R}$ is continuous.

Proof. Let $\theta^{(1)}$ and $\theta^{(2)}$ be two initial conditions in $\mathcal{D}_{0}$ at $t=1$. Then for the same admissible $\left\{u_{t}\right\}$ adapted to $\mathcal{F}_{t}$ with any given quantization scheme $\left\{r_{t}\right\}$, one may apply the comparison technique as in (Fleming and Rishel, 1975) to obtain

$$
\begin{aligned}
& \left|v\left(\theta^{(1)}\right)-v\left(\theta^{(2)}\right)\right|=\left|\inf _{u} J\left(\theta^{(1)}, u\right)-\inf _{u} J\left(\theta^{(2)}, u\right)\right| \\
& \leq \sup _{u}\left|J\left(\theta^{(1)}, u\right)-J\left(\theta^{(2)}, u\right)\right| .
\end{aligned}
$$


On the other hand, by the results in (Arapostathis and Marcus, 1990) it follows that

$$
\left|\theta_{t}^{(1)}-\theta_{t}^{(2)}\right| \leq C\left|\theta^{(1)}-\theta^{(2)}\right| \alpha^{t}
$$

for generic constants $C$ and $\alpha \in(0,1)$, where $\theta_{t}^{(i)}$ is given by (4) with initial condition $\theta^{(i)} \in \mathcal{D}_{0}$, $i=1,2$. For any admissible $\left\{u_{t}\right\}$, one can employ (9) together with $J(\theta, u)$ in (6) to get

$$
\left|J\left(\theta^{(1)}, u\right)-J\left(\theta^{(2)}, u\right)\right| \leq C_{1}\left|\theta^{(1)}-\theta^{(2)}\right|
$$

for some constant $C_{1}>0$, and then the continuity of the optimal cost follows readily.

Theorem 1. Under (H1), for $\theta \in \mathcal{D}_{0}$ the optimal control law $u$ is specified in a feedback form in terms of $\theta$. Specifically, each action $e_{i}^{u}, i \in I_{n}$, is associated with a convex action region contained in $\mathcal{D}_{0}$, and in the case $\theta_{t} \in \mathcal{D}_{0}$ lies in none of these action regions, the system should continue with its observations.

Proof. For each $e_{i}^{u}, i \in I_{n}$, its action region is defined as a subset of $\mathcal{D}_{0}$ by the relation:

$\mathcal{D}_{0} \supset \mathcal{A}_{i} \triangleq\left\{\theta \in \mathcal{D}_{0}: \min _{r} H\left(r, e_{i}^{u}, \theta\right)=\min _{r, u} H(r, u, \theta)\right\}$

with the convention that the minimization is taken w.r.t $r \in L_{d}$ and $u \in U$.

Now let us show that each $\mathcal{A}_{i}$ is a convex subset of $\mathcal{D}_{0}$. Letting $\theta_{1}, \theta_{2} \in \mathcal{A}_{i}$, it follows from concavity of the value function that

$$
v\left(\lambda \theta_{1}+(1-\lambda) \theta_{2}\right) \geq \lambda v\left(\theta_{2}\right)+(1-\lambda) v\left(\theta_{2}\right),
$$

where $\lambda \in[0,1]$. Notice that here one can establish concavity by the standard technique as in (Astrom, 1969) for finite horizon case and then taking limit to infinite horizon.

For any $\theta \in \mathcal{A}_{i}, i \in I_{n}$, the definition of $\mathcal{A}_{i}$ gives

$$
\begin{aligned}
& v(\theta)=\min _{r, u} H(r, u, \theta)=\min _{r} H\left(r, e_{i}^{u}, \theta\right) \\
= & \min _{r}\left[\theta^{T} c\left(\cdot, e_{i}^{u}\right)+\sum_{Y^{q}}\left|T\left(r, Y^{q}, e_{i}^{u}\right) \theta\right| v\left(\frac{T\left(r, Y^{q}, e_{i}^{u}\right) \theta}{\left|T\left(r, Y^{q}, e_{i}^{u}\right) \theta\right|}\right)\right] \\
= & \min _{r}\left[\theta^{T} c\left(\cdot, e_{i}^{u}\right)+v\left(e_{n+2}\right)\right] \\
= & \theta^{T} c\left(\cdot, e_{i}^{u}\right)+v\left(e_{n+2}\right),
\end{aligned}
$$

where the derivation has used the fact $\frac{T\left(r, Y^{q}, e_{i}^{u}\right) \theta}{\left|T\left(r, Y^{q}, e_{i}^{u}\right) \theta\right|}=$ $e_{n+2}$, and $\sum_{Y^{q}}\left|T\left(r, Y^{q}, e_{i}^{u}\right)\right|=1, i \in I_{n}$. Hence

$$
\begin{aligned}
& v\left(\lambda \theta_{1}+(1-\lambda) \theta_{2}\right) \geq \lambda v\left(\theta_{1}\right)+(1-\lambda) v\left(\theta_{2}\right) \\
= & {\left[\lambda \theta_{1}^{T}+(1-\lambda) \theta_{2}^{T}\right] c\left(\cdot, e_{i}^{u}\right)+v\left(e_{n+2}\right) . }
\end{aligned}
$$

On the other hand, it is obvious that

$$
\begin{aligned}
& v\left(\lambda \theta_{1}+(1-\lambda) \theta_{2}\right)=\min _{r, u} H\left(r, u, \lambda \theta_{1}+(1-\lambda) \theta_{2}\right) \\
\leq & \min _{r} H\left(r, e_{i}^{u}, \lambda \theta_{1}+(1-\lambda) \theta_{2}\right) \\
= & {\left[\lambda \theta_{1}+(1-\lambda) \theta_{2}\right]^{T} c\left(\cdot, e_{i}^{u}\right)+v\left(e_{n+2}\right) . }
\end{aligned}
$$

where the last equality is computed in the same manner as in (11), and therefore, (12) together with (13) yields

$$
\begin{aligned}
& v\left(\lambda \theta_{1}+(1-\lambda) \theta_{2}\right) \\
= & {\left[\lambda \theta_{1}+(1-\lambda) \theta_{2}\right]^{T} c\left(\cdot, e_{i}^{u}\right)+v\left(e_{n+2}\right) . }
\end{aligned}
$$

Thus, it follows that

$v\left(\lambda \theta_{1}+(1-\lambda) \theta_{2}\right)=\min _{r} H\left(r, e_{i}^{u}, \lambda \theta_{1}+(1-\lambda) \theta_{2}\right)$,

and $\lambda \theta_{1}+(1-\lambda) \theta_{2} \in \mathcal{A}_{i}$.

Proposition 2. If $\max _{i \in I_{n}} c_{1}(i) \leq \min _{i \in I_{n+1}} c_{0}(i)$, then the information state $e_{i} \in \mathcal{D}_{0}$ is in the action region $\mathcal{A}_{i}$ associated with the action $e_{i}^{u} \in U$.

Proof. Given $\theta=e_{i}, 1 \leq i \leq n$, if $c_{1}(i) \leq$ min $c_{0}(i)$, then clearly the action $u=e_{i}^{u} \in \bar{U}$ minimizes $\left.H(r, u, \theta)\right|_{\theta=e_{i}}$.

\subsection{Threshold policy for a single sensor}

With a single sensor, the detection framework using a network structure is meaningful since the sensor node does not have the data storage capacity and the past quantized measurements are preserved by the fusion center which can form more reliable estimates.

Proposition 3. Assume there is one sensor node and the condition in Proposition 2 holds, then the optimal control is specified as a threshold policy.

Proof. Since it has been shown that the action region for $e_{1}^{u} \in U$ is a convex set. In the single sensor node scenario, it can be concluded that the action set $\mathcal{A}_{1}$ is specified as an interval containing the end point $e_{1} \in \mathcal{D}_{0} \subset \mathbb{R}^{2}$. Hence the optimal control is determined as a threshold policy.

\subsection{Threshold policy for two sensors}

Consider two sensors with full coverage of the target transition, i.e., in the network there is not an outer site. In this case, the optimal control for the sensor nodes is still a threshold policy.

Proposition 4. Assume there are two sensor nodes for a two state target and the condition in Proposition 2 holds, then the optimal control at each node is specified as a threshold policy.

Proof. The proof is similar to that of Proposition 3 and is omitted here. 


\section{APPLICATION TO DISTRIBUTED CHANGE DETECTION}

In this section, a specific model for the uncontrolled Markov chain $X$ is considered as motivated by failure detection in large distributed systems consisting of subsystems. Suppose the underlying change or failure occurrence is associated with spatial locations each monitored by a sensor node with noisy measurements, and the sensors report local decisions to a fusion center. Typically, a system failure can be modelled by a geometric (or exponential in continuous time) distribution, and the tradition scheme is quickest detection with a tradeoff between the false alarm rate and detection delay; see (Kailath and Poor, 1998) (pp.2253-2254) and (Huang and Krishnamurthy, 2004). (Veeravalli, 2001) generalizes the traditional change detection in a sensor network where a set of i.i.d. measurements of sensor nodes switches all together from one distribution to another after disruption.

In our current formulation, based on sensor outputs, the fusion center not only needs to have a prompt decision about the change, but also needs to infer the associated site for the event. In other words, one needs to know when and where. Let us introduce the uncontrolled Markov chain $X$ with a transition probability matrix

$$
P=\left[\begin{array}{cccc}
1 & & \cdots & 0 \\
0 & 1 & \cdots & 0 \\
& & \vdots & \\
p_{n+1,1} & p_{n+1,2} & \cdots & p_{n+1, n+1}
\end{array}\right] .
$$

Let the initial "good" state of $X$ be $s_{n+1}$ (corresponding to the outer site in the target detection setting), and the change point $\tau$ be denoted by the time the state reaches from $s_{n+1}$ to other states each being absorbing. It is easily verified that the distribution of the change point is geometrically distributed. Let the sensor measurement be denoted by $Y_{i, t}$, and assume that indexed by $i$ and $t, Y_{i, t}$ are i.i.d with distribution $F_{1}$ conditioned on $t<\tau$. Conditioned on the event that the change occurs at site $\hat{i}$ and time $\tau=t$, then $Y_{\hat{i}, t}$, for all $t \geq \tau$ has distribution $F_{2} \neq F_{1}$. For all $i \neq \hat{i}$, each observation $Y_{i, t}$ is still described by the distribution $F_{1}$.

Apart from quantization optimization, here one can define the detection delay as well as false alarms by appropriately specializing the one stage cost in Section 2.3. Specifically, the fusion center decision should specify the location of the event and in the case of a mismatch, it is counted as a false alarm when delay, if applicable, is also taken into account. Such a distributed detection problem may be stated as a stopping problem and then converted into an optimal control problem, and one can further analyze the stopping region. The details are omitted here.

\section{REFERENCES}

Arapostathis, A. and S.I. Marcus (1990). Analysis of an identification algorithm arising in the adaptive estimation of Markov chains. Math. Control, Signals, and Systems 3, 1-29.

Aslam, J., Z. Butler, F. Constantin, V. Crespi, G. Cybenko and D. Rus (2003). Tracking a moving object with a binary sensor network. In: Proc. the 1st ACM Internat. Conf. Embedded Networked Sensor Systems. Los Angeles, CA. pp. 150-161.

Astrom, K.J. (1969). Optimal control of Markov processes with incomplete state information ii: the convexity of the loss function. J. Math. Analysis Applicat. 10, 403-406.

Bertsekas, D.P. (1995). Dynamic Programming and Optimal Control, Vol. 1,2. Athena Scientific. Belmont, MA.

Burr, S.A., J.E. Falk and A. F. Karr (1985). Integer prim-read solutions to a class of target defense probelms. Operations Res. 33, 726745 .

Chamberland, J.-F. and V.V. Veeravalli (2003). Decentralized detection in sensor networks. IEEE Trans. Signal Process. 51, 407-416.

Chong, C.-Y. and S.P. Kumar (2003). Sensor networks: evolution, opportunuties, and challenges. Proc. IEEE 91, 1247-1256.

Fleming, W.H. and R.W. Rishel (1975). Deterministic and Stochastic Optimal Control. Springer-Verlag. Berlin, Germany.

Huang, M. and S. Dey (2005). Distributed state estimation for hidden Markov models with dynamic quantization and rate allocation. In: Proc. 16th IFAC World Congress, Prague, Czeck.

Huang, M. and V. Krishnamurthy (2004). Risksensitive quickest time detection. In: Proc. 43rd IEEE Conf. Decision Control. Paradise Island, Bahamas. pp. 1748-1753.

Kailath, T. and H.V. Poor (1998). Detection of stochastic processes. IEEE Trans. Inform. Theory 44(6), 2230-2259.

Oh, S., L. Schenato and S. Sastry (2005). A hierarchical multiple-target tracking algorithm for sensor networks. In: Proc. Internat. Conf. Robotics and Automation. Barcelona, Spain.

Shiryayev, A.N. (1978). Optimal Stopping Rules. Springer. New York.

Veeravalli, V.V. (2001). Decentralized quickest change detection. IEEE Trans. Inform. Theory $47(4), 1657-1665$.

Zhao, F., Jie Liu, Juan Liu, L. Guibas and J. Reich (2003). Collabrative signal and information processing: an information-directed approach. Proc. IEEE 91(8), 1199-1209. 